\title{
Zurich Cementless Dual Mobility Cup for Canine Total Hip Prosthesis: Implant Characteristics and Surgical Outcome in 105 Cases
}

\author{
Otto I. Lanz ${ }^{1}$ Ida Forzisi ${ }^{2}$ Aldo Vezzoni² \\ ${ }^{1}$ Department of Small Animal Clinical Science, VA-MD Regional \\ College of Veterinary Medicine, Blacksburg, Virginia, United States \\ ${ }^{2}$ Clinica Veterinaria Vezzoni, Cremona, CR, Italy
}

\begin{abstract}
Address for correspondence Aldo Vezzoni, SCMPA, Dipl. ECVS, Clinica Veterinaria Vezzoni, Via delle Vigne 190, Cremona, CR 26100, Italy (e-mail: aldo@vezzoni.it).
\end{abstract}

Vet Comp Orthop Traumatol 2021;34:294-302.

\begin{abstract}
Keywords

- hip arthroplasty

- dual mobility cup

- cementless

- dog

- dislocation

Dislocation after total hip arthroplasty (THA) remains a troublesome complication, and a source of frustration for the owner and the surgeon. The dislocation rate of hip prostheses in dogs is reported to range from 4 to $15 \%$, representing the most common short-term complication. This is especially true in large and giant breed dogs, usually requiring revision surgery. With the increase in the number of THA being performed in veterinary surgery, reducing or preventing complications such as postoperative THA dislocation will be of paramount importance. The Zurich cementless dual mobility (DM) system allows impingement-free range of angulation of 80 to 132 degrees between the ceramic head and the polyether ether ketone (PEEK) cup when combined with the range of the PEEK cup in the outer metal cup. In this article, we review the use of the DM cup in THA in large and giant breed dogs, in terms of its history, biomechanics, outcomes and complications based on 105 cases.
\end{abstract}

\section{Introduction}

The concept of dual mobility (DM) was originally developed in the 1970s by Dr G. Bousquet for human total hip arthroplasty (THA) procedures, with the purpose of reducing the risk of dislocation of the hip prosthesis in the postoperative period. ${ }^{1}$ The original acetabular cup consisted of two bearings: a smaller ball inserted inside a larger polyethylene hemisphere, which articulated with a smooth metal surface. Primary movement occurs at the inner bearing with the DM cup. The secondary articulation, between the liner and the acetabular shell, engages during activities that exceed the normal range of motion (ROM) of the hip, when the femoral component impinges on the rim of the liner. ${ }^{2}$

Dislocation following a THA continues to be the major complication associated with the THA procedure, with rates of luxation ranging from 1.9 to $5.8 \%$ in humans. ${ }^{3,4}$ In 2010 , the Australian Joint Registry reported that dislocations accounted for $14.4 \%$ of THA revision procedures. ${ }^{5}$ The dislocation rate of hip prostheses in dogs is reported to range from

received

June 23, 2020

accepted after revision

January 15, 2021

published online

March 31, 2021

4 to $15 \%$, representing the most common short-term complication. ${ }^{6-15}$ This dislocation rate complication is especially true in large and giant breed dogs, and necessitates revision surgery, leading to further costs to the owner, prolonged recovery times, or, in the worst-case scenario, explantation. ${ }^{13,15}$ The rate of dislocation when using a DM cup prosthesis in the initial THA procedure in people is reported to be between 0 and $1.1 \%{ }^{16-18}$ In the veterinary field, the only documented use of a DM cup is reported by Guillaumot and colleagues. ${ }^{19,20}$ In his second publication, a cemented DM cup prosthesis was implanted in dogs, and no dislocation was experienced in 50 consecutive cases. ${ }^{20}$ The overall rate of dislocation following a THA depends on several aspects, such as appropriate positioning of the implants, orientation and offset of the acetabular and femoral implants and the preservation of the local soft tissues. ${ }^{3,21,22}$ It has been shown that a large diameter head can decrease the rate of luxation. ${ }^{23,24} \mathrm{~A}$ larger diameter head improves stability by not only affecting the jump distance, which is the degree of

(c) 2021. Thieme. All rights reserved. Georg Thieme Verlag KG,

Rüdigerstraße 14,

70469 Stuttgart, Germany
DOI https://doi.org/ 10.1055/s-0041-1725015. ISSN 0932-0814. 
lateral translation of the centre of the femoral head required before dislocation occurs, but also reducing impingement by altering the head-to-neck ratio, thus increasing the ROM before impingement and dislocation. ${ }^{25}$ There may be decreased wear when using a DM cup, as there are two interfaces, and thus less friction and sliding at each interface. This has been proven in the laboratory, but not yet clinically. ${ }^{26,27}$ Long-term in vivo wear studies are needed to determine wear in comparison with standard acetabular cups. In humans, DM cups are used in complex primary or revision THA. ${ }^{28-30}$ THA for displaced fractures of the femoral neck has a higher rate of dislocation than THA performed for osteoarthritis. ${ }^{30,31}$ In dogs, the DM cup is potentially useful in cases with higher risk of dislocation, as is currently seen in cases with chronic hip luxation, nonreparable femoral head fractures, unsuccessful femoral head and neck excisional arthroplasty, large and giant breed dogs and young, giant breed dogs with already severe hip dysplasia with hip laxity (luxoid hips). The cementless hip prosthesis is now widely used in both the human and veterinary fields, especially in young patients, where a biological fixation by osseointegration guarantees a longer duration of the implants. $6,14,15,32-34$ Therefore, the development of a cementless DM cup was motivated by the need to have a DM cup for the Zurich cementless prosthetic system to be used in dogs with a known increased risk of dislocation following a THA procedure. The adoption of carbon fibre ring (CFR)/polyether ether ketone (PEEK, Evonik, Germany) cup insert instead of highly crosslinked polyethylene since January 2016 in the Zurich system allowed the outer bearing of the DM cup to be made of PEEK, which is more resistant to wear when compared with polyethylene. ${ }^{35}$

The purpose of this study is to review the use of the Zurich cementless DM system in THA in terms of its history, biomechanics, outcomes and complications based on 105 cases.

\section{Materials and Methods}

\section{Implant Characteristics}

The Zurich cementless DM system has two main components, the cup and the head (-Fig. 1A). The cup is composed of an outer and an inner shell, both made from a titanium alloy. The outer shell is perforated and coated by porous titanium and hydroxyapatite for rapid bone ingrowth and increased compliance. The inner shell's articulation surface is coated by titanium nitrate. The head of the DM articulation has two components. The outer head component is made of PEEK with a carbon fibre reinforced PEEK ring (PEEK/CFRPEEK) on the inside. The outer surface of the PEEK head component articulates with the titanium nitrate surface of the cup and the carbon fibre reinforced PEEK ring inside it articulates with the second component of the head, which is made of ceramic. The ceramic (VERILOX, CeramTec, Germany) head is preassembled with the PEEK head by snap-fitting that allows for free rotation between the two but also provides strong retention in the axial direction. The outer head component has a nominal diameter of $25 \mathrm{~mm}$ and the ceramic head has a $19 \mathrm{~mm}$ diameter.

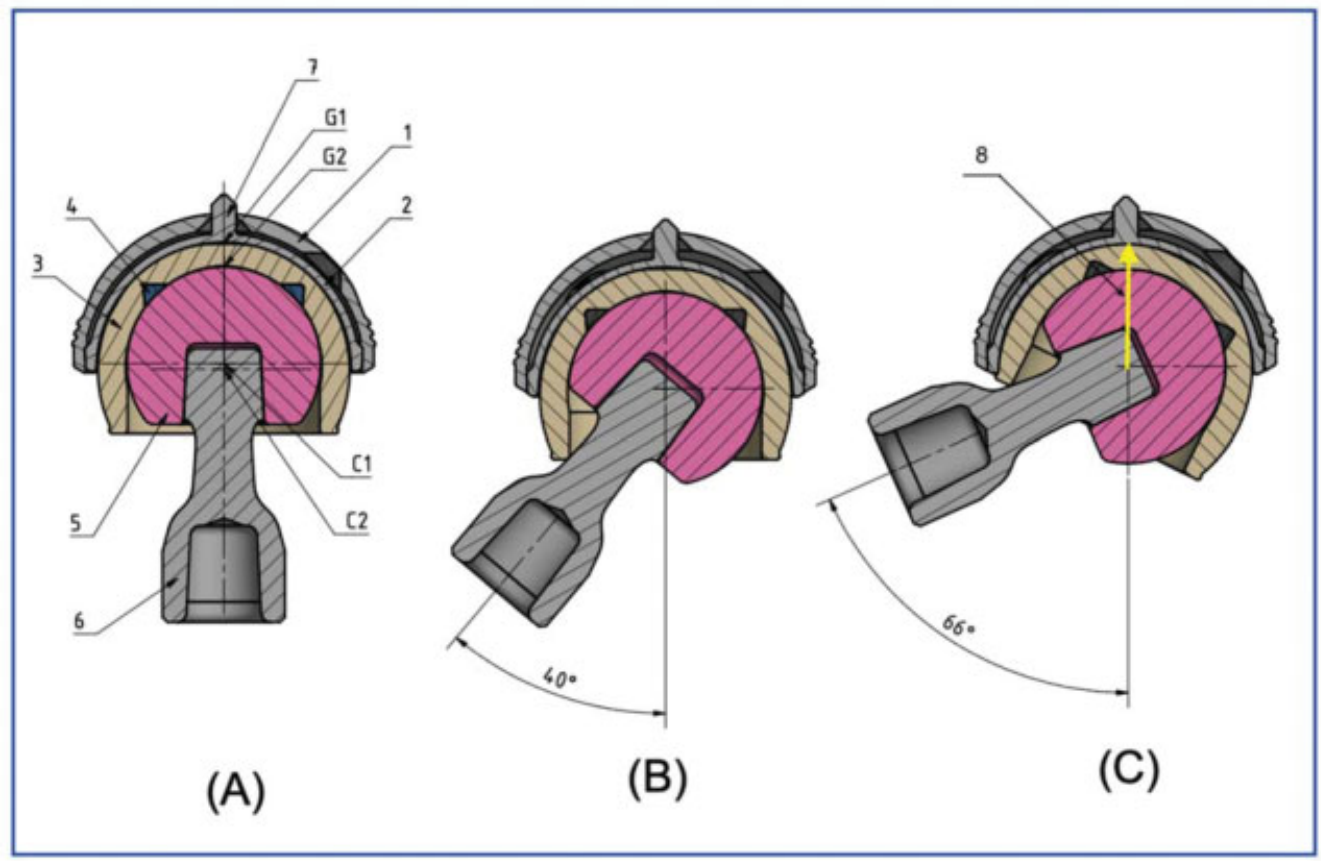

Fig. 1 Technical drawings (complete description in the text). (A,1-4) The four components of the Zurich dual mobility cup system. (A, 5) Ceramic (Ceravet) head of the articulation. (A, 6) Neck of the prosthesis. (A, 7) Spike guided into its receiving hole in the outer shell for concentric assembly of the shells, by either the manufacturer or in surgery for the revision-style cup; G1, small gap at the outer surface of the polyether ether ketone (PEEK) head component; G2, small gap at the primary articulation surface between ceramic head and carbon fibre ringPEEK ring. (B) Eighty degrees of impingement-free range of angulation between ceramic head and PEEK head. (C) Impingement-free range increased to 132 degrees when combined with range of PEEK head in the outer metal cup; (C, 8) The jumping distance (yellow arrow) is the degree of lateral translation of the femoral head centre required for the hip to dislocate. 
The articulation (-Fig. 1A) is attached to the neck of the prosthesis via a standard conical coupling. The spike is guided into its receiving hole in the outer shell facilitating concentric assembly of the shells, by either the manufacturer or in surgery for the revision-style cup. The inner titanium nitrate coated surface of the inner shell is spherical in shape. The outer surface of the PEEK component is aspherical in shape leaving a small gap, G1, at the pole resulting in an annular contact at the articulation.

This articulation geometry is shown to reduce the wear rate by approximately a factor of three, when compared with sphere-in-sphere articulation with a polar contact. ${ }^{36}$ At the primary articulation surface, the spherical ceramic head makes an annular contact with the CFR-PEEK ring leaving a small gap, G2, at the pole. The ceramic head is snap-fitted into the PEEK head by the manufacturer. The centre of rotation $\mathrm{C} 1$, between the ceramic head and the PEEK head, is positioned deeper into the cup than the centre of rotation $\mathrm{C} 2$, between the PEEK head and the cup. This theoretically provides a slight bias in forces tending to align the PEEK cup over the average joint force. The friction moment between the ceramic head and the PEEK head is smaller than that between the PEEK head and the cup, so most of the motion is expected to happen at the ceramic head-CRF-PEEK ring interface, which results in the lowest wear of known articulations, except for perhaps ceramic-ceramic pairing. ${ }^{34}$ The impingement-free range of angulation between the ceramic head and the PEEK head of 80 degrees ( - Fig. 1B) is increased to 132 degrees (-Fig. 1C) when combined with the range of the PEEK head in the metal cup. The ceramic head diameter is $19 \mathrm{~mm}$; the nominal diameter of the PEEK head is $25 \mathrm{~mm}$; the nominal outer cup diameter is $29.5 \mathrm{~mm}$ and $32.5 \mathrm{~mm}$; and the nominal neck diameter is $5.5 \mathrm{~mm}$. During extreme flexion or extension of the hip, the femoral neck moves the PEEK head inside the acetabular component, which has a highly polished articulating surface of titanium nitrate (-Fig. 2).

\section{Surgical Technique}

The technique of performing a primary THA using the DM cup is similar to that of a standard Zurich THA. Full exposure of the acetabulum needs to be obtained prior to reaming the acetabulum, with the femur retracted in a caudoventral direction following excision of the head and neck. The outer shell of the cup is implanted between 40 and 45 degrees of lateral opening, and 15 and 20 degrees of retroversion. Lateral opening is evaluated visually with the impaction bar aligned to 45 degrees, provided that the pelvis is in true lateral position, and checked with an image intensifier. Due to the variability in pelvis conformation in dogs of different breeds, the cup retroversion is aimed to follow the anatomy of the acetabulum, centring the cup between the cranial and caudal pillars of the acetabulum. To impact the DM cup, the impactor for the 26.5 revision cup is used. The femur is prepared in the standard fashion, paying particular attention to the preoperative planning measurements and preservation of the surrounding soft tissues, ensuring that appropriate offset is obtained. Fixation of the cup when performing a revision THA may require fixation with screws. In such an instance, a disassembled DM cup is

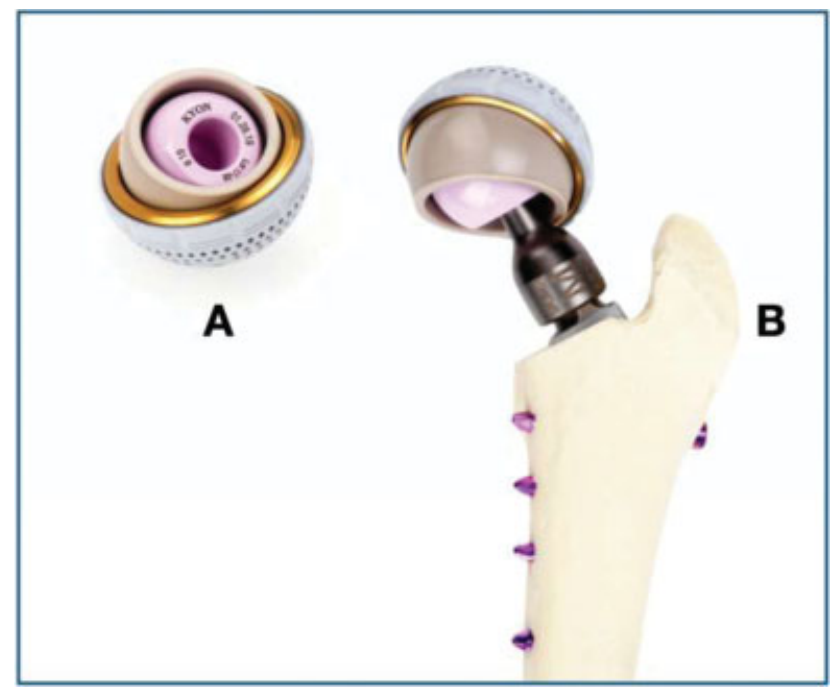

Fig. 2 (A) Dual mobility cup and dual mobility head. (B) The neck inserted into head of the dual mobility head. The neck is first inserted into the ceramic head followed by insertion of the peg of the femoral prosthesis into the neck.

used to fix the outer shell with 2.0 or $2.4 \mathrm{~mm}$ cortical screws (-Fig. 3A and B). The inner shell is impacted next inside the outer shell. The inner shell is aligned in a perfectly coaxial direction by the spike in the pole of the inner shell, which in turn aligns with a receptacle in the centre of the outer shell. Before implanting the femoral stem, the clearance between the cup and the femoral neck osteotomy is checked. This is performed by inserting a $23 \mathrm{~mm}$ trial cup into the DM cup (outer shell) while keeping the positional bar vertical and contacting the femoral osteotomy. The femur is simultaneously retracted with a Hohmann retractor or with pointed reduction forceps on the trochanter with distal distraction. A clearance of $\sim 2 \mathrm{~cm}$ should be obtained to allow reduction of the prosthesis with a medium neck, which is indicated in large dogs where a $29.5 \mathrm{~mm}$ or a $32.5 \mathrm{~mm}$ cup is required. When the clearance is less than $2 \mathrm{~cm}$, the femoral osteotomy is modified in a distal direction, and the clearance is re-evaluated. Reduction of the DM head requires a different technique compared with the reduction of a standard cup and $16 \mathrm{~mm}$ or $19 \mathrm{~mm}$ heads. Because of the overall larger diameter of $25 \mathrm{~mm}$ (ceramic head plus CFR-PEEK head), the jumping distance necessary for reduction is greater when compared with the standard method, making reduction more difficult (-Fig. 1C). To simplify the reduction, it is recommended that the preassembled head and CFR-PEEK head be impacted on the correct neck first. The neck, head and CFR-PEEK head are inserted into the cup. The femur is then levered, so that the peg of the femoral prosthesis is reduced into the neck. To summarize, the correct order for ease of reduction is to first impact the preassembled DM head (ceramic head plus CFR-PEEK head) on the appropriate neck, and then insert both inside the cup. The femur is retracted laterally and distally with a pointed bone holding forceps fixed at the greater trochanter, and the peg of the femoral stem is guided until it enters the recess of the neck. To complete the insertion of the peg of the femoral stem inside the recess of the neck, a narrow Hohmann retractor, inserted caudally to the acetabulum, is used to lever the femur laterally 


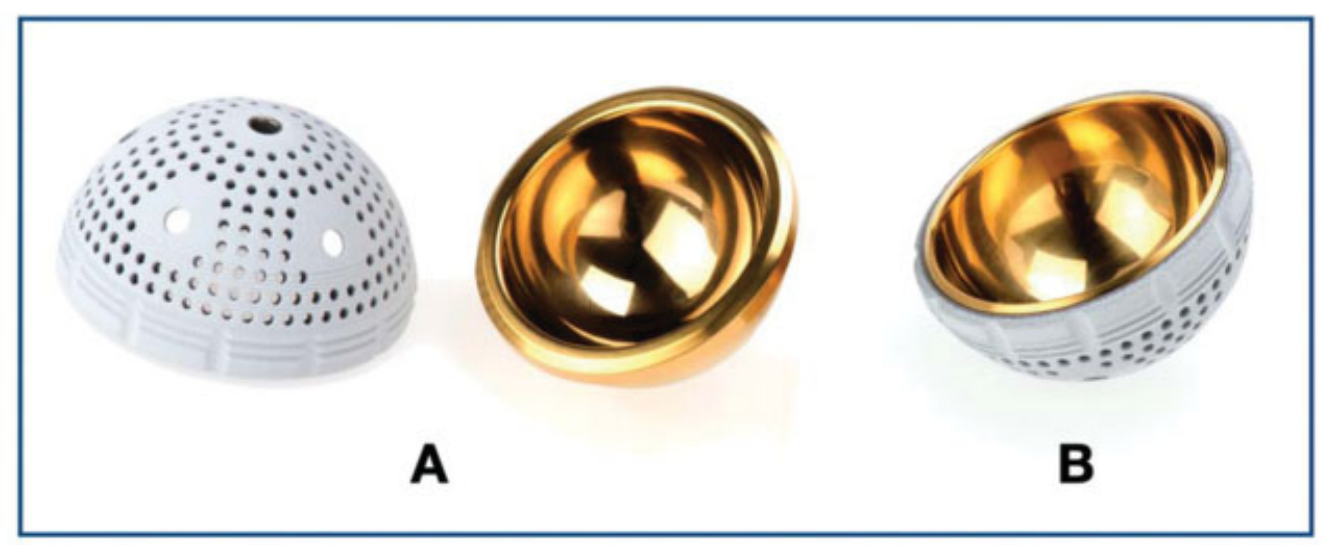

Fig. 3 Dual Mobility Cup. (A) The inner shell (gold in colour) is impacted inside the outer shell (white in colour). (B) The inner shell is aligned in the coaxial direction by a spike in the pole of the inner shell, aligning the centre of the outer shell.

and distally, allowing the peg of the femoral prosthesis to enter the recess of the neck. With this method, accidental entrapment of soft tissues inside the DM cup during reduction is avoided. The usual tests for stability of the reduction are performed, followed by the closure of the anatomical layers.

Standard radiographic views are performed to evaluate the implant orientation and position: ventrodorsal view with the hip flexed to evaluate the deepness of insertion of the cup, lateral view of the pelvis with superimposed ilial bodies and tuber ischii to measure the angle of lateral opening and to evaluate retroversion of the cup, mediolateral view of the femur with the stifle positioned at 90 degrees to evaluate the stem anteversion, and an oblique view of the pelvis with the acetabular cup viewed fully laterally (radiographic beam tangent to the equator of the cup) with the femur perpendicular to the pelvis and slightly externally rotated, which allows the X-ray beam to be perpendicular to the screws of the stem. The latter most radiographic projection is obtained with fluoroscopic guidance for accurate positioning.

\section{Indications for Dual Mobility Cup}

There are no extensive published data on the use of DM cups in dogs, except for the aforementioned publication by Guillaumot and colleagues. ${ }^{20}$ However, it is the authors' experience that large and giant breed dogs have an increased risk of dislocation following a THA procedure compared with smaller dogs using standard cups and heads. The DM cup is currently available in two dimensions, 29.5 and $32.5 \mathrm{~mm}$, because of the space required for the ceramic head plus the CFR-PEEK inner liner. Due to these limited sizes, its use is indicated only in large and giant breed dogs, for whom those sizes of cups are appropriate. In those dogs, especially when affected by preoperative joint instability, the risk of luxation should be greatly reduced when compared with the published rate of luxation following a THA procedure without using the DM protheses.

\section{Results}

A total of 105 THA were performed using the Zurich cementless total hip system with the DM cup between April 2018 and March 2020 at the Clinica Veterinaria Vezzoni, Cremona, Italy, by the same surgeon (AV). There were 53 males and 34 females for a total of 87 dogs, with 18 dogs having bilateral THA performed.

Median body weight was $43 \mathrm{~kg}$ (range: $30-64 \mathrm{~kg}$ ), and median age was 37 months (range: 6-116 months). Breeds included are as follows: Bernese Mountain dog $(n=14)$, German Shepherd dog $(n=14)$, Rottweiler $(n=14)$, Cane Corso $(n=8)$, Labrador retriever $(n=7)$, Mixed breed $(n=6)$, Newfoundland $(n=5)$, Leonberger $(n=3)$, Maremma Shepherd $(n=3)$, Central Asia Shepherd $\operatorname{dog}(n=2)$, Dogue de Bordeaux $(n=2)$ and 1 each for the following 9 different breeds - Akita Inu, Alaskan Malamute, Broholmer, Bull Mastiff, Golden retriever, Bobtail, Rhodesian Ridgeback, Russian terrier and Swiss Shepherd dog. The 29.5 cup was used in 97 cases (92\%) and the 32.5 in 8 cases ( $8 \%$ ). A short neck was used in 13 cases (12\%), a medium neck in 86 cases (82\%), a long neck in 4 cases ( $4 \%$ ) and an $\mathrm{x}$-long neck in 2 cases ( $2 \%$ ). The radiographic and clinical follow-up examinations had a median follow-up time of 5 months with a range of 2-24 months and were available in 85 of 87 (98\%) dogs (-Figs. 4 and 5). Two cases in this study using the DM cup were performed as revisions of previously performed Zurich cementless THA surgeries that later dislocated. The first case involved a 15-month-old male intact Dogue de Bordeaux that dislocated 2 months following surgery (26.5 mm acetabular cup with a $19 \mathrm{~mm}$ head). A $29.5 \mathrm{~mm}$ DM cup was used to revise this case and at the 6month follow-up evaluation, no postoperative complications were noted and the implants radiographically appeared stable. The second case involved a 3-year-old female intact Newfoundland that had a previously performed Helica THA that failed 4 months following surgery due to failure of the Helica stem. Initially the Helica stem was solely replaced with Kyon stem and Helica $18 \mathrm{~mm}$ neck/head unit suitable for Kyon stem peg, but the prosthesis dislocated 15 days following surgery. A second revision was performed with a $29.5 \mathrm{~mm}$ DM cup. Radiographs performed 4 months following the second revision revealed no abnormalities.

One case involving a Cane Corso had a Zurich cementless THA procedure (26.5 $\mathrm{mm}$ cup and $19 \mathrm{~mm}$ head) performed in the left hip, which dislocated in the postoperative period and was revised by replacing the medium neck with a long neck. A Zurich cementless THA using a $29.5 \mathrm{~mm}$ DM cup was later 

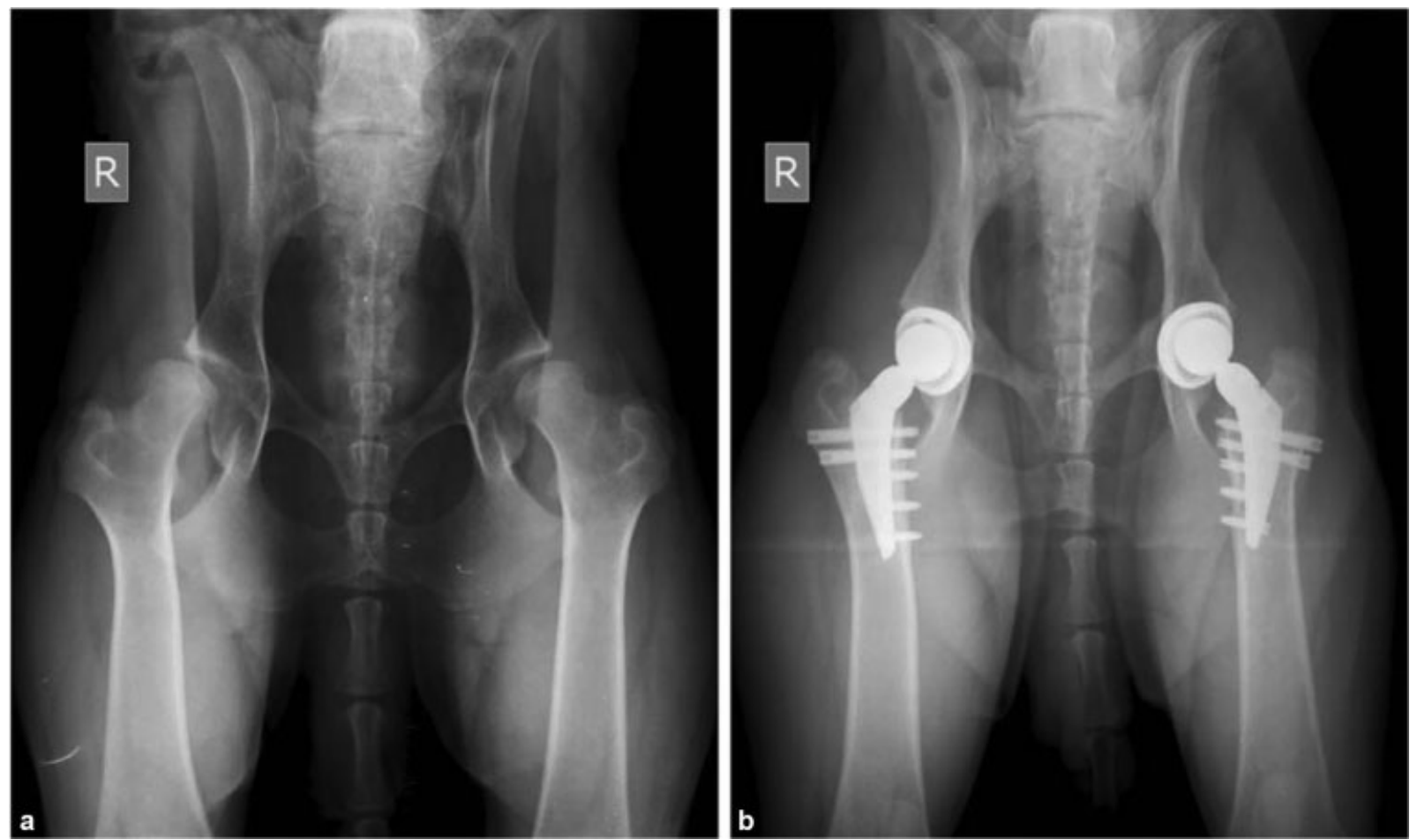

Fig. 4 Pelvic radiographs of a 29-month-old male Bernese Mountain dog, $39.6 \mathrm{~kg}$, with bilateral total hip replacement using dual mobility cups. Left hip was done at 11 months of age and the right hip at 14 months of age.

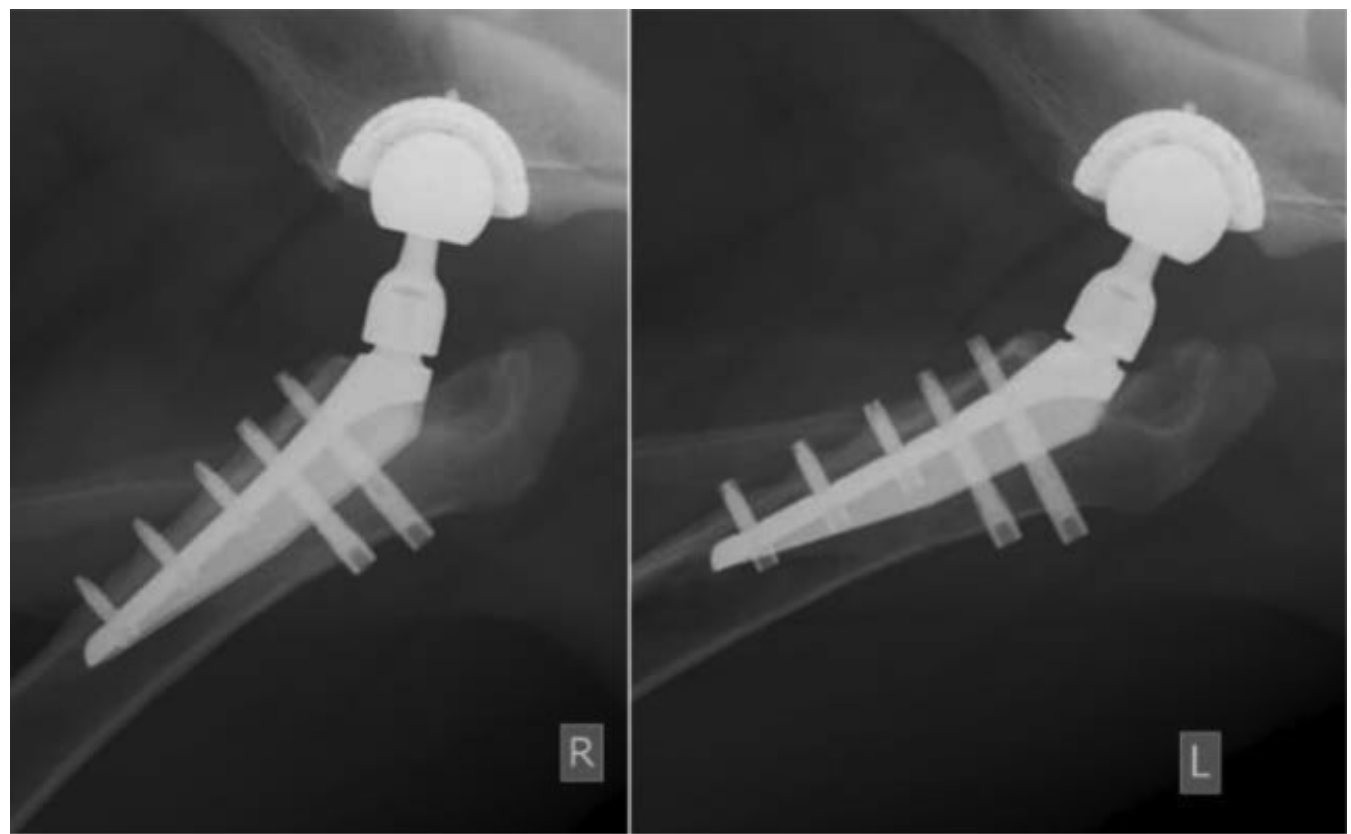

Fig. 5 Same dog as in - Fig. 4. Follow-up with oblique pelvic views tangent to the implants: left after 18 months and right after 15 months reveal normal bilateral total hip replacements.

performed in the right hip with no postoperative complications encountered.

Four cases involved dogs with traumatic hip luxations. This group consisted of a: 3-year-old male mongrel dog (38 kg) after failed attempts of using an Ehmer sling, 15month-old female intact Bernese Mountain dog (35 kg) 1 month after a failed Slocum sling, 5-year-old female Bernese Mountain dog (40 kg) with a chronic traumatic hip luxation and a 9-year-old female Golden retriever $(46 \mathrm{~kg})$ with a chronic traumatic hip luxation.

The final group of dogs was categorized with conditions known to have a high rate of postoperative dislocation following THA surgery. This consisted of 7 cases with capital physeal fractures, 1 case with an unsatisfactory femoral head and neck excisional arthroplasty, and 22 cases with luxoid hips in large breed dogs. 
There was one dislocation that occurred 27 months following surgery in a 1-year-old male intact Bernese Mountain Dog that had a left THA using a $29.5 \mathrm{~mm}$ DM cup, medium neck, and a large stem. There was evidence of caudal ventral subluxation observed on pelvic frog view radiograph (normal in the extended radiograph) 9 months following surgery but the dog was asymptomatic. The dog became acutely lame at 27 months following surgery and radiographs at this time revealed dorsal dislocation of the implant. This case was successfully revised inserting a new $25 \mathrm{~mm}$ DM head, and a long neck.

One dislocation of the prosthesis was encountered ( 1 of 105 cases) in the luxoid group of dogs. No other complications were noted in the short-term follow-up period in the remaining dogs. There were no notable changes in any of the remaining dogs (104 cases) on the follow-up radiographs with regard to wear or observed changes in the dimensions of the components of the DM cup or head in any of the cases. As well, there was no evidence of femoral loosening or screw loosening in any of the cases (105 cases).

\section{Discussion}

While THA is an extremely successful operation in dogs as in people, dislocation remains one of the most frustrating complications. DM articulation lowers the risk of dislocation by creating a favourable femoral head-to-neck ratio, which decreases impingement. ${ }^{2,20,30,37}$

There are several mechanisms responsible for the increased stability afforded by the DM components. Several authors have noted that stability is improved with an increased femoral head-to-neck ratio, a concept known as the McKee-Farrar principle, which increases the jump distance required for the femoral head to separate from the acetabular component. 2,24,33,38,39 The design of the DM cup provides a favourable head-to-neck ratio by allowing movement between the metal component and the outer polyethylene or PEEK bearing, the size of which determines the effective diameter of the femoral head. The increased jump distance decreases the risk of luxation when the femoral head or neck impinges on the acetabular component. The incidence of impingement is also decreased by increasing the ROM of the dual-bearing head within the acetabular component. With the Zurich cementless DM cup, the ROM is 145 degrees compared with 135 degrees with the standard cup and $19 \mathrm{~mm}$ head. The smaller, inner head provides most of the effective movement.

The most severe complication described in humans is intraprosthetic dislocation, which differs from the more common extra-articular dislocation. Intraprosthetic dislocation is also referred to as retentive failure, which is unique to DM cups and originally described in humans. ${ }^{30,39}$ These types of dislocation are extremely difficult to reduce using closed techniques, and require an open reduction. The newer models of DM cups have vastly decreased the rates of dislocation in comparison with the original design. Most reports of the newer designs describe a rate of luxation of $0 \%{ }^{16,29,40}$ Intraprosthetic dislocation was not observed in any of our cases, since the ceramic head and CFR-PEEK head are preassembled by the manufacturer. One case of wear and breakage of the polyethylene inner liner (15 mm internally and $20 \mathrm{~mm}$ externally) of a cemented DM cup was described by Vedrine and colleagues in a young hunting English Setter 4 months after surgery. ${ }^{41}$ According to the authors, the use of a long neck with flanges led to the failure of the ultrahigh molecular weight polyethylene, promoting abnormal wear of the component during extreme ROM. This may explain the unusual complication encountered. However, as the CFR-PEEK head in Zurich DM cup degrades due to internal wear, its ability to constrain the ceramic head may decrease, which increases the risk of intraprosthetic dislocation. To reduce this risk, the carbon fibre reinforced ring is manufactured inside the PEEK inner liner, where the load of the ceramic head is mainly concentrated ${ }^{35}$ (-Fig. 6). Also, the abnormal wear between the PEEK head and the metal acetabular component could lead to wear of the PEEK head, but longterm follow-up of these cases will be required to evaluate this possibility.

Abnormal wear causes the production of wear particles, which may lead to failure in bone integration to the prosthesis. Aseptic loosening may be associated with particulate debris caused by degradation of bearing surfaces, but an association between aseptic loosening and modern DM components with highly crosslinked polyethylene is not described in humans. ${ }^{26,30,42,43}$ Another theoretical concern

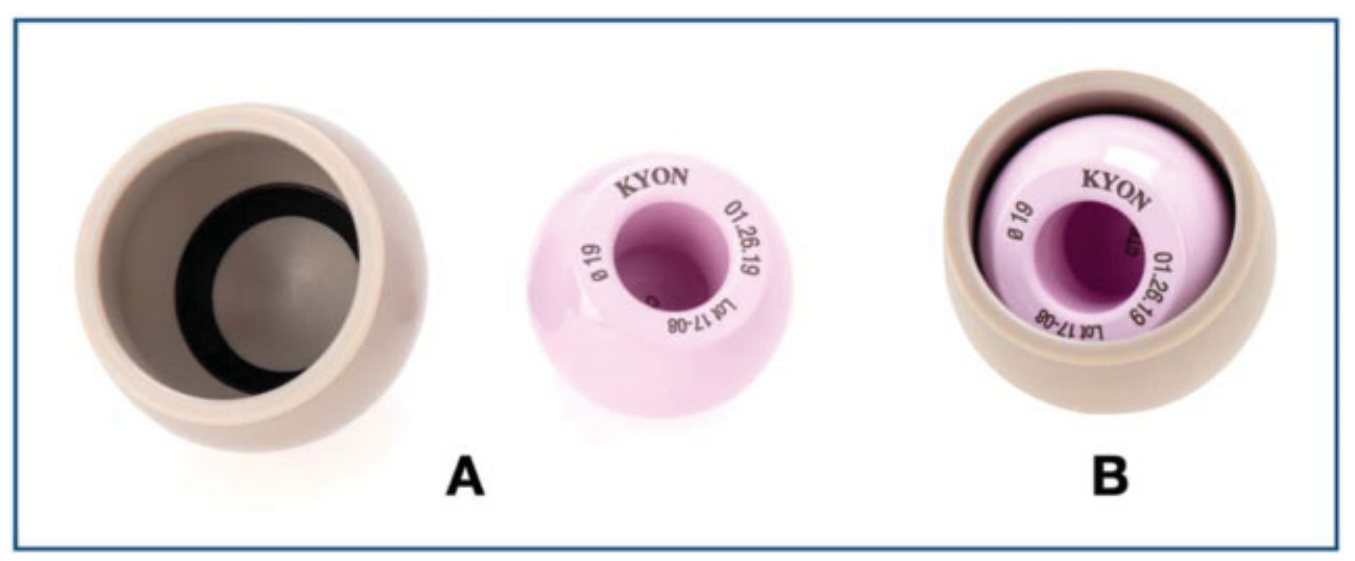

Fig. 6 (A) The inner components of the dual mobility system consist of the ceramic head (VERILOX) and carbon fibre ring-polyether ether ketone outer head. (B) The head components are preassembled by the manufacturer and are inserted after the outer titanium shell is impacted in the acetabulum. 
is that PE wear may be proportional to the surface area of the articulation, which is higher in DM cups compared with single-bearing design cups. ${ }^{26,42}$

A DM cup by nature allows wear to occur on both surfaces of the head (inside and outside), and on the articulating head, which potentially could increase the wear of polyethylene. However, in vitro studies demonstrate that PEEK is more resistant to wear compared with polyethylene. ${ }^{35}$ The polyethylene wear in dogs leads to reactive bone resorption around the implants, and can ultimately be seen as early as 3 to 4 years after a THA procedure. ${ }^{15,44,45}$ This is termed 'wear disease'. PEEK could also lead to aseptic loosening and 'wear disease'; however, this complication has not been observed in our cases with PEEK liners, even after 4 years following THA procedures using DM cups (personal communication).

Is it possible that a $25 \mathrm{~mm}$ single-bearing primary THA head provides the same stability as a DM THA? A correlation between the size of the femoral head and stability is reported in the human orthopaedic literature. ${ }^{24,30}$ In one retrospective study comparing DM cups and single-bearing $36 \mathrm{~mm}$ heads in primary THA for patients at high risk for luxation, the rates of luxation and revision in the DM cup patients were much lower when compared with patients receiving a single-bearing $36 \mathrm{~mm}$ head. ${ }^{25}$

A potential disadvantage for some, but not all, DM cups used in humans is that they lack screw holes. ${ }^{28,30-32}$ As with all monolithic cups, the inability to visualize the floor of the acetabulum during the impaction process and not being able to anchor the outer shell with screws may compromise overall fixation of the DM cup. This is less likely to be a cause of failure in primary THA procedures than with revision procedures, where there may be significant bone loss associated with the acetabulum. The Zurich cementless DM cup is available also as a revision cup, with the two components of the shell to be assembled in surgery after the outer shell is secured to the acetabulum using 2.0 or $2.4 \mathrm{~mm}$ cortical screws. In our cases, one Zurich cementless DM revision cup $(29.5 \mathrm{~mm})$ was used in a Dogue de Bordeaux, which luxated after having a Zurich cementless $26.5 \mathrm{~mm}$ cup and $19 \mathrm{~mm}$ head. The revision DM cup was stable at the 6-month follow-up evaluation.

Large and giant breed dogs, especially young dogs ( $<1$ year of age) with very unstable hips (luxoid hips), were shown in a previous study evaluating the standard Zurich cementless hip system to have an incidence of dislocation of $8.5 \%$ at 2 months (personal communication). In our preliminary experience with the Zurich cementless DM cup, we observed one dislocation of the prosthesis in a dog with a known increased risk of dislocation, but no other dogs in this group had a dislocation. Guillaumot and colleagues reported zero cases of dislocation with the cemented DM cup in the postoperative period. ${ }^{20}$ In our cases, dislocation was observed 27 months following surgery in one dog with luxoid hips. This case dislocated due to repeated episodes of subluxation between the PEEK/ CFR head and the cup of the DM system. This repeated subluxation leads to deformity of the PEEK/CFR liner causing the inner ceramic head to be locked inside the PEEK/CFR liner, leading to the loss of mobility within the DM cup. The loss of full mobility of the DM cup leads to impingement and dislocation of the prosthesis. Soft tissue entrapment can also lead to loss of mobility of DM cups leading to dislocation. Careful attention not to entrap soft tissues in the DM cup during reduction of the prosthesis is imperative to prevention of dislocation. The authors recommend preassembling the neck to the head, already inserted in the PEEK/CFR liner, prior to inserting the head and PEEK/CFR liner in the outer shell. Reduction is then performed by toggling the femoral peg into the neck, completing the reduction.

In the available follow-up of our cases (103 THA procedures in 85 dogs), no other complication was reported. Nevertheless, longer-term follow-ups are required to establish the longevity of this new cementless DM cup, which represents a limitation of this clinical study.

\section{Conclusion}

Dual mobility cups may be an alternative to standard singlebearing cups in dogs reported to be at high risk for luxation in both revision and primary arthroplasty. The complication rate, including dislocation, is relatively low in the cases reported in this study $(1 / 103,1 \%)$.

\section{Authors' Contributions}

O.L. and A.V. conceptualized and designed the study. I.F. and A.V. acquired, analyzed and interpreted the data. All authors drafted, revised and approved the submitted manuscript and are publically accountable for relevant content.

\section{Conflict of Interest}

None declared.

\section{Acknowledgments}

The authors would like to thank Slobodan Tepic and Stephen Bresina who developed this Dual Mobility System, Reto Hitz who did the drawings of - Fig. 1 and Kyon $(\mathrm{CH})$, producer of the Zurich Cementless THR, who provided the photos of the implants.

\section{References}

1 Bousquet GDF, Debiesse JL, Girardin P, Relave M, Israeli A. The ceramic coated cementless total hip arthroplasty: basic concepts and surgical technique. J Orthop Surg Tech 1985;1:15-28

2 Plummer DR, Haughom BD, Della Valle CJ. Dual mobility in total hip arthroplasty. Orthop Clin North Am 2014;45(01):1-8

3 Lewinnek GE, Lewis JL, Tarr R, Compere CL, Zimmerman JR. Dislocations after total hip-replacement arthroplasties. J Bone Joint Surg Am 1978;60(02):217-220

4 Woo RY, Morrey BF. Dislocations after total hip arthroplasty. J Bone Joint Surg Am 1982;64(09):1295-1306

5 Australian Orthopaedic Association National Joint Replacement Registry. 2010. Accessed July 29, 2015 at: https://aoanjrr.dmac. adelaide.edu.au/

6 Guerrero TG, Montavon PM. Zurich cementless total hip replacement: retrospective evaluation of 2 nd generation implants in 60 dogs. Vet Surg 2009;38(01):70-80

7 Hayes GM, Ramirez J, Langley Hobbs SJ. Does the degree of preoperative subluxation or soft tissue tension affect the 
incidence of postoperative luxation in dogs after total hip replacement? Vet Surg 2011;40(01):6-13

8 Olmstead ML, Hohn RB, Turner TM. A five-year study of 221 total hip replacements in the dog. J Am Vet Med Assoc 1983;183(02): 191-194

9 Conzemius MG, Vandervoort J. Total joint replacement in the dog. Vet Clin North Am Small Anim Pract 2005;35(05): 1213-1231

10 Schulz KS. Application of arthroplasty principles to canine cemented total hip replacement. Vet Surg 2000;29(06):578-593

11 Forster KE, Wills A, Torrington AM, et al. Complications and owner assessment of canine total hip replacement: a multicenter internet based survey. Vet Surg 2012;41(05):545-550

12 Kidd SW, Preston CA, Moore GE. Complications of porous-coated press-fit cementless total hip replacement in dogs. Vet Comp Orthop Traumatol 2016;29(05):402-408

13 Hummel DW, Lanz OI, Werre SR. Complications of cementless total hip replacement. A retrospective study of 163 cases. Vet Comp Orthop Traumatol 2010;23(06):424-432

14 Fitzpatrick N, Law AY, Bielecki M, Girling S. Cementless total hip replacement in 20 juveniles using $\mathrm{BFX}^{\mathrm{TM}}$ arthroplasty. Vet Surg 2014;43(06):715-725

15 Vezzoni L, Vezzoni A, Boudrieau RJ. Long-term outcome of Zurich cementless total hip arthroplasty in 439 cases. Vet Surg 2015;44 (08):921-929

16 Vielpeau C, Lebel B, Ardouin L, Burdin G, Lautridou C. The dual mobility socket concept: experience with 668 cases. Int Orthop 2011;35(02):225-230

17 Boyer B, Philippot R, Geringer J, Farizon F. Primary total hip arthroplasty with dual mobility socket to prevent dislocation: a 22-year follow-up of 240 hips. Int Orthop 2012;36(03):511-518

18 Combes A, Migaud H, Girard J, Duhamel A, Fessy MH. Low rate of dislocation of dual-mobility cups in primary total hip arthroplasty. Clin Orthop Relat Res 2013;471(12):3891-3900

19 Guillaumot P, Autefage A, Palierne S, Dembour T, Chancrin JL. Dual mobility canine total hip prosthesis: implant characteristics and surgical procedure. Vet Comp Orthop Traumatol 2012;25(06): 506-510

20 Guillaumot P, Autefage A, Dembour T, Chancrin JL. Outcome and complications after dual mobility total hip replacement: fifty cases with a minimum of six months clinical and radiographic follow-up. Vet Comp Orthop Traumatol 2012;25(06):511-517

21 Sanchez-Sotelo J, Berry DJ. Epidemiology of instability after total hip replacement. Orthop Clin North Am 2001;32(04):543-552

22 Dyce J, Wisner ER, Wang Q Olmstead ML. Evaluation of risk factors for luxation after total hip replacement in dogs. Vet Surg 2000;29 (06):524-532

23 Chandler DR, Glousman R, Hull D, et al. Prosthetic hip range of motion and impingement. The effects of head and neck geometry. Clin Orthop Relat Res 1982;(166):284-291

24 Berry DJ, von Knoch M, Schleck CD, Harmsen WS. Effect of femoral head diameter and operative approach on risk of dislocation after primary total hip arthroplasty. J Bone Joint Surg Am 2005;87(11): 2456-2463

25 Haughom BD, Plummer DR, Moric M, Della Valle CJ. Is there a benefit to head size greater than $36 \mathrm{~mm}$ in total hip arthroplasty? J Arthroplasty 2016;31(01):152-155

26 Epinette JA, Béracassat R, Tracol P, Pagazani G, Vandenbussche E. Are modern dual mobility cups a valuable option in reducing instability after primary hip arthroplasty, even in younger patients? J Arthroplasty 2014;29(06):1323-1328

27 Adam P, Farizon F, Fessy MH. [Dual articulation retentive acetabular liners and wear: surface analysis of 40 retrieved polyethylene implants]. Rev Chir Orthop Repar Appar Mot 2005;91(07): 627-636 (In French)

28 Civinini R, Carulli C, Matassi F, Nistri L, Innocenti M. A dualmobility cup reduces risk of dislocation in isolated acetabular revisions. Clin Orthop Relat Res 2012;470(12):3542-3548

29 Vasukutty NL, Middleton RG, Matthews EC, Young PS, Uzoigwe CE, Minhas TH. The double-mobility acetabular component in revision total hip replacement: the United Kingdom experience. J Bone Joint Surg Br 2012;94(05):603-608

30 Matsen LK, Hozack WJ. Outcomes of dual mobility components in total hip arthroplasty. A systematic review of the literature. Bone Joint J 2016;98-B(1, Suppl A):60-63

31 Langlais FL, Ropars M, Gaucher F, Musset T, Chaix O. Dual mobility cemented cups have low dislocation rates in THA revisions. Clin Orthop Relat Res 2008;466(02):389-395

32 Li M, Glassman A. What's new in hip replacement. J Bone Joint Surg Am 2019;101(18):1619-1627

33 Marcellin-Little DJ, DeYoung BA, Doyens DH, DeYoung DJ. Canine uncemented porous-coated anatomic total hip arthroplasty: results of a long-term prospective evaluation of 50 consecutive cases. Vet Surg 1999;28(01):10-20

34 Bergh MS, Budsberg SC. A systematic review of the literature describing the efficacy of surgical treatments for canine hip dysplasia (1948-2012). Vet Surg 2014;43(05):501-506

35 Brockett C, John G, Williams S, Isaac G, Fisher J. Wear of Ceramicon-Carbon Fibre Reinforced PEEK Hip Replacements. Orthopaedic Proceedings Vol. 94-B, No. SUPP_XL 2018

36 Tepic S, Bresina S, Hintner M, Kaddick C. Reduced wear of a UHMWPE THR liner with modified contact geometry. 53rd Annual Meeting of the Orthopaedic Research Society, Poster 1805

37 McArthur BAND, Nam D, Cross MB, Westrich GH, Sculco TP. Dualmobility acetabular components in total hip arthroplasty. Am J Orthop 2013;42(10):473-478

38 McKee GK, Watson-Farrar J. Replacement of arthritic hips by the McKee-Farrar prosthesis. J Bone Joint Surg Br 1966;48(02): 245-259

39 Lachiewicz PF, Watters TS. The use of dual-mobility components in total hip arthroplasty.J Am Acad Orthop Surg 2012;20(08):481-486

40 Hamadouche $M$, Arnould $\mathrm{H}$, Bouxin B. Is a cementless dual mobility socket in primary THA a reasonable option? Clin Orthop Relat Res 2012;470(11):3048-3053

41 Vedrine B, Guillaumot P, Chancrin JL. Dislocation of a dual mobility total hip replacement following fracture of the polyethylene liner. Vet Comp Orthop Traumatol 2016;29(03):259-264

42 Stulberg SD. Dual poly liner mobility optimizes wear and stability in THA: affirms. Orthopedics 2011;34(09):e445-e448

43 Netter JD, Hermida JC, Chen PC, Nevelos JE, D'Lima DD. Effect of microseparation and third-body particles on dual-mobility crosslinked hip liner wear. J Arthroplasty 2014;29(09):1849-1853

44 Vezzoni L, Montinaro V, Vezzoni A. Use of a revision cup for treatment of Zurich cementless acetabular cup loosening. Surgical technique and clinical application in 31 cases. Vet Comp Orthop Traumatol 2013;26(05):408-415

45 Nesser VE, Kowaleski MP, Boudrieau RJ. Severe polyethylene wear requiring revision total hip arthroplasty in three dogs. Vet Surg 2016;45(05):664-671 